\title{
Spirulina (Arthrospira) industry in Inner Mongolia of China: current status and prospects
}

\author{
Yun-Ming Lu • Wen-Zhou Xiang • Yong-Huang Wen
}

Received: 27 January 2010 /Revised and accepted: 16 June 2010/Published online: 7 July 2010

(C) The Author(s) 2010. This article is published with open access at Springerlink.com

\begin{abstract}
This paper outlines an investigation on current situation of Spirulina (Arthrospira) industry in Inner Mongolia, an internal region of China with temperate continental climate. More than 20 Spirulina plants have been established in Inner Mongolia since 2001, most of which are located at Wulan Town in the Ordos Plateau. By the end of 2009, the total annual production of Spirulina in the Ordos Plateau surpassed $700 \mathrm{t}(\mathrm{dw})$, which account for ca. $80 \%$ of the total productivity of Inner Mongolia, and ca. $20 \%$ of China. Besides abundant solar radiation and enough freshwater favorable for Spirulina production, the three technical strategies contribute to the prosperity and success of Spirulina industry in the region: (1) reducing the cost or investment by overall advantages of rich local natural resources with low cost for Spirulina production, such as alkaline lakes, coal, electricity, and sandy land; (2) controlling the culture temperature and to avoid contamination by building plastic greenhouses on raceway ponds, (3) reducing investment by simplifying the construction of the ponds and the greenhouses. As the result, the growth period of Spirulina has been prolonged from about 120 to about 165 days, the cost of Spirulina has decreased by 25 $30 \%$, and the quality of products has been enhanced
\end{abstract}

This paper was presented at the 7th Asia Pacific Congress on Algal Biotechnology, New Delhi 2009.

Y.-M. Lu $(\bowtie) \cdot$ Y.-H. Wen

Shenzhen Ludebao Health Food Co., Ltd.,

Shenzhen 518040, China

e-mail: Luyunming@263.net

W.-Z. Xiang $(\bowtie)$

South China Sea Institute of Oceanology,

Chinese Academy of Sciences,

Guangzhou 510301, China

e-mail: xwz@scsio.ac.cn substantially. Inner Mongolia is expected to become the largest base for Spirulina production not only in China, but also in the world in the near future.

Keywords Spirulina production · Alkaline lake ·

Temperate climate $\cdot$ Plastic greenhouse $\cdot$ Inner Mongolia

\section{Introduction}

Spirulina (Arthrospira), a blue-green alga containing unusual high amounts of balanced proteins and a wide range of abundant nutraceuticals, is one of the most promising microalgae in the market since the success of its commercial production in open raceway ponds in the 1980s (Voushak and Richmond 1988). Spirulina cultivation plants have sprung up in China since the early 1990s. By the mid 1990s, China has become the biggest country in Spirulina production in the world ( $\mathrm{Li}$ and Qi 1997; Wu et al. 1998). Total production of Spirulina in China was about $2,500 \mathrm{t}(\mathrm{dw})$ in $2006(\mathrm{Hu}$ 2007). According to our observation and investigation, up to 2009, 3,500 t (dw) of Spirulina have been produced.

Inner Mongolia has a mid temperate climate with an annual average temperature of $6.4^{\circ} \mathrm{C}$ which greatly limits the growth of Spirulina (Qiao et al. 2001). Although there were several reports examining the feasibility of industrial production of Spirulina in temperate climates earlier (Jiménez et al. 2003), most Spirulina plants at present are located at tropical and sub-tropic area in the world since Spirulina is thermophilic with optimal growth temperature between 35 and $38^{\circ} \mathrm{C}$ ( $\mathrm{Li}$ and Qi 1997; Belay 1997; Wu et al. 1998).

Consequently, it is of significance that large-scale commercial production in Inner Mongolia has been achieved. 
This paper analyses how the Spirulina industry was established successfully in Inner Mongolia, and discusses the prospects of local Spirulina industry. Suggestions for sustainable development of commercial production of Spirulina in this region in the future are made.

\section{History and present status}

Local species of Spirulina were discovered in alkaline lakes in the Ordos Plateau of Inner Mongolia in 1995, and at least three species, including Spirulina (Arthrospira) platensis and two other novel species of Arthrospira were discovered and isolated ( $\mathrm{Li}$ et al. 2003; Hu 2007). In 1997, a pilot-scale experiment was conducted to probe the feasibility of commercial production of local Spirulina species in Inner Mongolia (Qiao et al. 2001), but no further progress was reported. In 2001, the first commercial Spirulina plant with open raceway ponds was established in Ordos Plateau (Hu 2007).

In the early stage (2001-2003) of Spirulina production in Ordos Plateau, the culture in open raceway ponds was affected by the low temperatures and sand storm which resulted in low quality and productivity of products. The majority of Spirulina produced in this stage was sold with low profit as low-grade animal feeds. Because of the technical improvement in Spirulina production after 2003, the total annual production of Spirulina increased rapidly. The total annual production of Spirulina, as well as total ponds area in Ordos in the past 4 years is presented in Fig. 1. The major manufacture plants in Ordos City and their production data in 2008 are listed in Table 1. It shows that the productivity of Spirulina in Ordos during production period is close to or even higher than that of sub-tropic or tropic areas in China (Li et al. 2003; Wu et al. 1998).

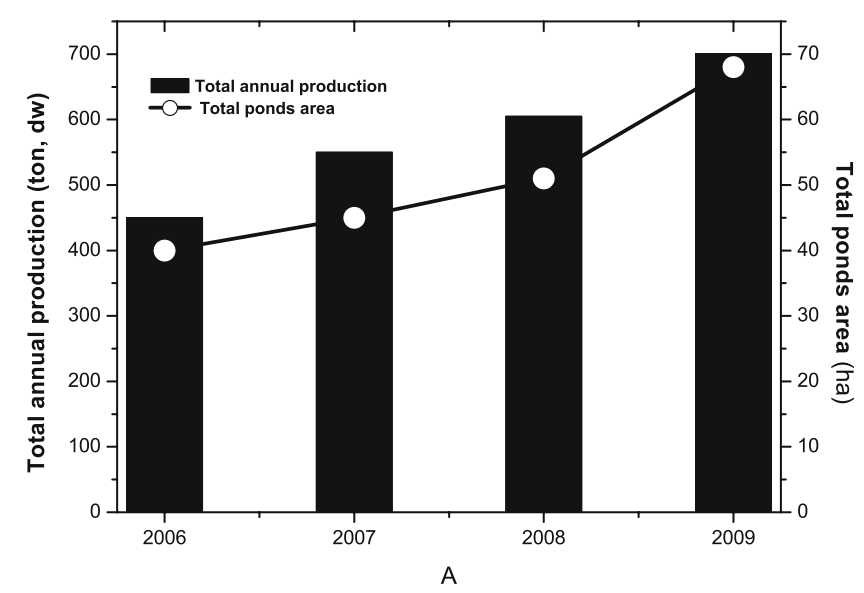

Fig. 1 Total annual production of Spirulina and total pond area in the last 4 years in Ordos city
Up to now, more than 20 companies have produced Spirulina in different areas of Inner Mongolia, including Ordos City, Alxa Meng and Bayannaoer Meng. Most Spirulina plants in Inner Mongolia are located at Wulan Town in the Ordos Plateau, southwest Inner Mongolia. Total ponds area of at least 13 companies, covering more than 70 ha, is used for large-scale cultivation of Spirulina. Based on our investigation, in 2009, the total annual production of Spirulina exceeded $700 \mathrm{t}(\mathrm{dw})$ in Ordos, about $80 \%$ of Spirulina produced in Inner Mongolia and about $20 \%$ of that in China.

It has been indicated that several native Spirulina (Arthrospira) species isolated in Inner Mongolia have potential for commercial production ( $\mathrm{Li}$ et al. 2003; $\mathrm{Hu}$ 2007). However, according to our investigation, another two common foreign species, S. platensis from Lake Chad, Africa, and S. maxima from lake Texcoco, Mexico, both of which are thermophilic, are used widely in present commercial production in Inner Mongolia.

Since both the quality and productivity have been enhanced in recent years, most of Spirulina produced in Inner Mongolia is sold as food, food additive, high-grade animal feeds, and bioactive substances with phycocyanin and polysaccharides involved. The alga powder as human food or animal feed was the main product sold earlier, however recently, with an increasing need for Spirulina tablets as health foods whether from China or overseas, many Spirulina plants in Inner Mongolia have begun to produce Spirulina tablets, bringing more unit profit than alga powder. Meanwhile, some high-value products, such as phycocyanin, polysaccharides and polypeptides extracted from Spirulina, have been developed, but the market is very limited. One company produces and sells fresh biomass of Spirulina in the form of oral liquid (Table 1).

In fact, Inner Mongolia has become one of the most important bases for commercial production of Spirulina in China. Compared with other places in China, the cost of Spirulina production in this region has decreased by 25 $30 \%$, which has become the main impetus for development of Spirulina industry.

What has happened in recent years for Spirulina production in Inner Mongolia? The following information and discussion about the advantages, disadvantages, and technical strategies for Spirulina production in Inner Mongolia attempts to answer this question.

\section{The advantages, disadvantages, and strategies}

First of all, due to a large number of alkaline lakes are located in Inner Mongolia, bicarbonate with a very low price can be used for Spirulina cultivation. In Ordos plateau alone, there are 65 alkaline lakes in over a $1-\mathrm{km}^{2}$ area, with 
Table 1 Main Spirulina plants in Ordos and their production profile in 2008

\begin{tabular}{|c|c|c|c|c|}
\hline Company & $\begin{array}{l}\text { Ponds area } \\
\text { (ha) }\end{array}$ & $\begin{array}{l}\text { Annual production } \\
\text { (Ton, dw) }\end{array}$ & $\begin{array}{l}\text { Productivity } \\
\left(\mathrm{gm}^{-2} \mathrm{~d}^{-1}\right)^{\mathrm{a}}\end{array}$ & Products sold \\
\hline Shuangfengbao Greenalgae Co., Ltd. & 9.30 & 132 & 8.61 & Alga powder as food and animal feeds \\
\hline Luyuan microalgae Co., Ltd. & 6.20 & 57 & 5.57 & $\begin{array}{l}\text { Alga powder, oral liquid, phycocyanin, } \\
\text { polysaccharides }\end{array}$ \\
\hline Erdos Jiali Srirulina Co., Ltd. & 6.50 & 83 & 7.74 & Alga powder, tablets, polypeptide \\
\hline Luweibao Spirulina Bioeng. Co., Ltd. & 4.75 & 58 & 7.41 & Alga powder, tablets, capsule \\
\hline Mengjian Spirulina Industry Co., Ltd. & 3.00 & 39 & 7.88 & Alga powder as food and animal feeds \\
\hline Bulonghu Bioeng. Develop. Co., Ltd. & 4.09 & 38 & 5.64 & Alga powder, capsule \\
\hline Derong algal Industry Co., Ltd. & 3.80 & 57 & 9.09 & Alga powder, tablets, \\
\hline Lufeng Bioeng. Co., Ltd. & 2.84 & 28 & 5.97 & Alga powder, tablets, \\
\hline Weida Spirulina Industry Co., Ltd. & 4.52 & 65 & 8.72 & Alga powder, tablets \\
\hline Huayitai Spirulina Co., Ltd & 5.50 & 48 & 5.29 & Alga powder, tablets \\
\hline
\end{tabular}

${ }^{a}$ The productivity was calculated on the basis of average annual production period, 165 days

a total area of all alkaline lakes of $189 \mathrm{~km}^{2}$ (Qiao et al. 2001). Generally, according to our analysis and recorded data, high concentration of alkali exists in most alkaline lakes in the forms of carbonate and bicarbonate. In some lakes, alkali concentration is over $260 \mathrm{mM}$. An important technical strategy in many plants is to utilize alkaline mother liquor which provides bicarbonate for Spirulina growth. Through application of the new strategy, the cost of bicarbonate, calculated on the basis of solid form, has decreased to 19-43 US\$ per ton, much lower than that of commercial bicarbonate (food grade), at about 190 US\$ per ton.

Secondly, the short rainy season, long daylight hours and the abundant solar energy in this region are optimal natural conditions for Spirulina production. The average annual precipitation is between 280 and $360 \mathrm{~mm}$ and average annual daylight sunshine is $3,000 \mathrm{~h}$. Total annual solar radiation is $5,730-5,930 \mathrm{MJ} \mathrm{m}^{-2}$ in this region, which will provide plenty of solar energy for growth of Spirulina.

Thirdly, with rich coal resources, the price of electricity supplied by coal-fired power plants for Spirulina production in Inner Mongolia is much lower than other places in China. Moreover, coal could be used in spray-drying, which could save $50-80 \%$ of the drying cost of Spirulina, compared with other places in coastal or middle region of China. Encircled by the Great Bend of the Yellow River, the Ordos plateau provides plenty of clean surface and underground fresh water.

Finally, since the climate of western Inner Mongolia like Ordos Plateau is semi-arid, the sandy land around alkaline lakes, mostly desert, is very cheap. Therefore, due to the land price, along with simplified construction of culture ponds which are underlain by plastic liner after the ground is leveled, compacted, and shaped as the structure of raceway ponds with the help of simple and cheap framework, the cost of investment to construct a Spirulina plant has decreased significantly compared with other places in the developed eastern coastal region of China.

However, considering specific disadvantages for Spirulina production in this region, some special measures are taken. The average annual temperature is $6.4^{\circ} \mathrm{C}$ with a long period of frost days. Low temperature is the key obstacle for Spirulina cultivation. There would be only a period of $\sim 120$ days for Spirulina growing in open raceway ponds in this region if no measures are taken to control the culture temperature. Meanwhile, since strong wind and sandstorms often occur here, sand, dust and possibly microbes will contaminate Spirulina cultures in open ponds and reduce the quality of the product. In order to overcome these problems, one of the most important strategies in Spirulina cultivation in this region is to build plastic greenhouses over the raceway ponds (Fig. 2, 3). The plastic greenhouse is made of a cheap framework (mixture of steel and bamboo or wood) and transparent plastic membrane. The plastic greenhouse enhances the culture temperature significantly (Fig. 4) and prolongs the growth period up to an average of 165 days with a maximum of up to 180 days. The maximum daily temperature of the culture is over $30^{\circ} \mathrm{C}$ and up to $42^{\circ} \mathrm{C}$ from May to September, and over $20^{\circ} \mathrm{C}$ and up to $39^{\circ} \mathrm{C}$ except for a several days in April or October.

With the help of the thermo-proof plastic greenhouse, the diurnal variation of culture temperature is $3-5^{\circ} \mathrm{C}$ in most days of growth period, which are optimal for Spirulina production. However, in the early stage (April) of the year, "seeds" management (incubation and expansion) is very important for Spirulina production of the whole season and is constrained easily by extra low temperature during cloudy or rainy days in April (Fig. 4). In some plants, temperature of "seeds" culture in night is maintained by an 
Fig. 2 A typical Spirulina plant with plastic greenhouse at Wulan Town, Ordos City; Photographed by Yong-Huang Wen

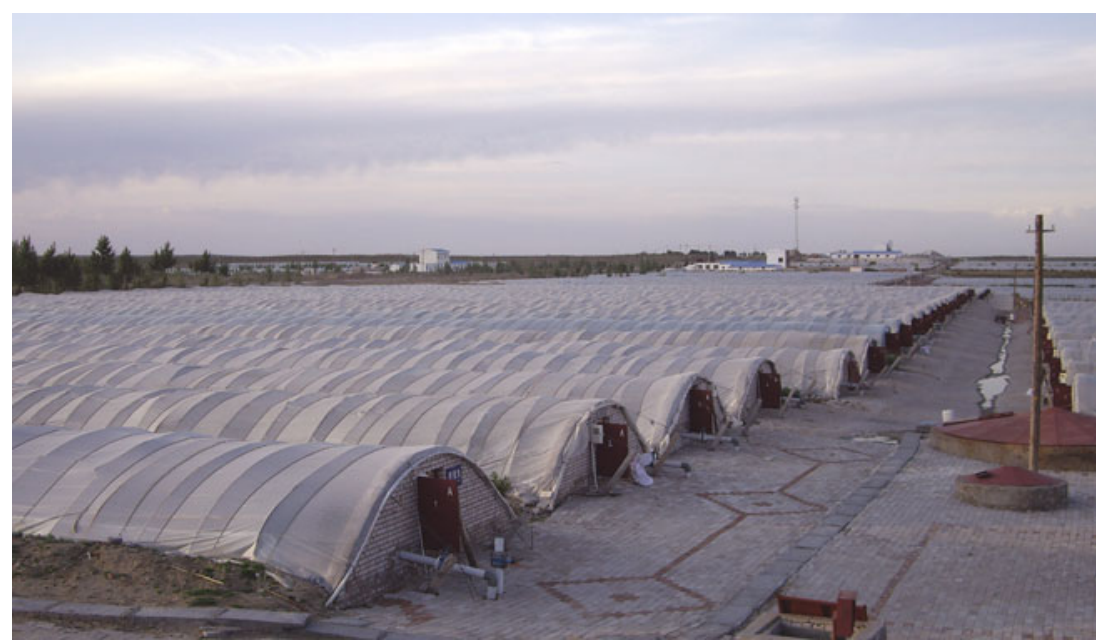

additional thermo-proof layer made of synthetic fiber cotton and covering the greenhouse of "seed" ponds. The "seed" culture is also electrically heated in several plants in this stage, which maintains culture temperature at over $25^{\circ} \mathrm{C}$ all day. By using these combined methods of temperature control, S. platensis or S. maxima, which are thermophilic algae (Vonshak 1997), can be used in this area as the "seeds" of large-scale cultivation.

By utilizing all the advantages and technical strategies above, the total annual production has increased sharply, the quality has been improved, and the production cost of Spirulina has decreased in recent years in Inner Mongolia.

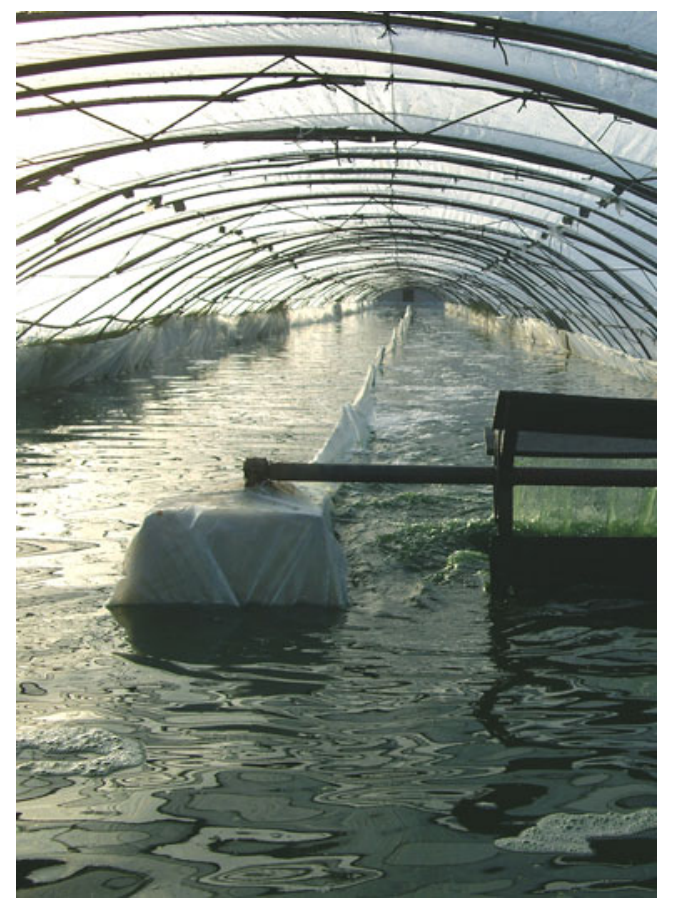

Fig. 3 Simplified inner structure of the culture pond and the greenhouse for Spirulina cultivation at Wulan Town, Ordos City. Photographed by Yong-Huang Wen
After offsetting the extra cost of transportation, investment in the construction of greenhouses and others, the cost of Spirulina production here is $25-30 \%$ lower than elsewhere (ca 3-4 US\$ kg-1) in China. Now, the quality of Spirulina produced in Ordos Plateau is comparable with that produced in other places in China, meeting the requirement of food safety not only in China, but also in the EU.

Consequently, a novel model for commercial production of thermophilic Spirulina with large-scale was formed in temperate attitude, which would be of great significance both for China and for other places in the world.

\section{Problems, prospects, and suggestions}

There are noticeable differences in Spirulina production among different companies (Table 1). According to our

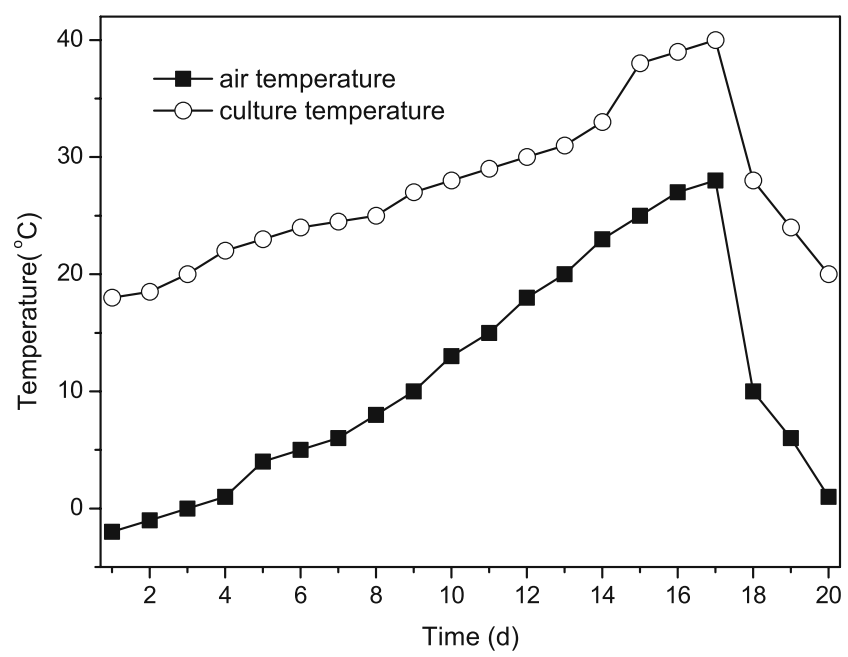

Fig. 4 Effect of plastic greenhouse on temperature controlling of the Spirulina culture. Outdoor air temperature and maximum culture temperature is recorded on 13:00 pm in consecutive 20 days in April, 2009 in a Spirulina plant at Wulan Town, Ordos City 
investigation, many technical factors, including stain or species, "seed" management, control of culture conditions, harvest of biomass and cyclically reusing the culture medium, contribute to this. There are many differences in these aspects among different companies, for instance, different companies use different sources of "seed" (strains) of Spirulina, and different models of "seed" management are applied in different seasons by different producers. Some companies do not pay enough attention to control temperature overnight when "seed" expansion begins in April and others maintain the "seed" under suitable scale and optimum temperature even in winter. There are also many differences in other technical aspects of Spirulina production.

All these differences indicate that there is no common technical standard in Spirulina production among companies in this region currently. Therefore, we suggest that a general technical standard be set up as the guide for the producers in this region, forming protocols on four technical aspects in Spirulina production: (1) strain or species and "seed" management; (2) control of temperature, cell density, dissolved oxygen, $\mathrm{pH}$, and speed of paddle wheel; (3) strategy for addition of $\mathrm{CO}_{2}$ and nutrients; (4) strategy for biomass harvest and reuse of old medium. Among these, "seed" management and temperature control are particularly important for Spirulina production in temperate Inner Mongolia. Meanwhile, standard for quality controlling in Spirulina production and processing, for instance, GMP, ISO9001 or HACCP, should be established in order to promote sustainable development of Spirulina industry.

In Inner Mongolia, Ordos City alone has a total land area of $20,064 \mathrm{~km}^{2}$ with a large proportion of sandy land. The local government and Spirulina producers have an ambitious plan to enlarge the scale of the Spirulina industry to a great extent. A special industry zone for Spirulina production was established earlier at Wulan Town in Ordos City, where a Spirulina project has been initiated and aims to reach an annual output of 3,000 t (dw) in a few years. A 1,000,000-t plan for Spirulina production has also been sketched out. Considering the nutritional and therapeutic function, the widespread application of Spirulina as human food, animal feeds, and other high valuable products (Belay 2002; Gershwin and Belay 2008), and the advantage of realizing low-cost production of Spirulina by using above-mentioned strategies in this region, we believe that this would not be an unreachable dream for the local government and industries.

For the purpose of better development of the Spirulina industry in Inner Mongolia in the future, suggestions on technical innovation and improvement of the Spirulina industry are presented as follows.

Coal and other natural energy resources could be used better in Spirulina production, for instance, wind energy is very rich in this region, it would be economical to run paddle-wheels by using wind. In the process of energy generation or Spirulina drying, $\mathrm{CO}_{2}$ and residual heat could be used as a carbon resource or applied to temperature/pH control in Spirulina cultivation. Selection and application of low-temperature-adapted strains would also be very important for Spirulina production. Besides raceway ponds with greenhouse cover, design of novel closed photobioreactors with effective temperature maintenance, high productivity, and reasonable cost would be an alternative approach to prolong the growth period of Spirulina. Development of high value products as well as cheap products with a large market could be encouraged. It could be expected that though the improvement and innovation of technology in the near future, Inner Mongolia will become the largest base for Spirulina production not only in China, but also in the world.

Acknowledgment This work is supported by the National Natural Sciences of China projects (grant no.: 40776087, 30471330) and key project of the Chinese Academy of Sciences (Grant No. KZCX2-YWJS207). We thank De-Sheng Lu, Xiang Li and Fen-Fen Liu for checking the English.

Open Access This article is distributed under the terms of the Creative Commons Attribution Noncommercial License which permits any noncommercial use, distribution, and reproduction in any medium, provided the original author(s) and source are credited.

\section{References}

Belay A (2002) The potential application of Spirulina (Arthrospira) as a nutritional and therapeutic supplement in health management. The J Am Nutraceut Assoc 5(2):26-49

Belay A (1997) Mass culture of Spirulina outdoors - the Earthrise Farms experience. In: Vonhask A (ed) Spirulina platensis (Arthrospira): Physiology, Cell-Biology and Biotechnology. Taylor \& Francis, London, UK, pp 131-158

Gershwin ME, Belay A (eds) (2008) Spirulina in human nutrition and health. CRC, Boca Raton

$\mathrm{Hu}$ HJ (2007) Prospects on large-scale cultivation of Arthrospira (Spirulina) by using alkaline lakes in China. In New frontiers of phycology-Proceedings on the 312nd Xiangshan Science Conference, Nov. 7-9th, 2007, Beijing, China, pp.21

Jiménez C, Cossío BR, Labella D, Niell FX (2003) The feasibility of industrial production of Spirulina (Arthrospira) in Southern Spain. Aquaculture 217:179-190

Li BS, Qiao C, Tian XY (2003) Potential to development Spirulina plantensis with alkaline lakes in Arodos Plateau. Plant Mag 06:18-20

Li DM, Qi YZ (1997) Spirulina industry in China: present status and future prospects. J Appl Phycol 9:25-28

Qiao C, Li BS, Zeng ZQ (2001) Alkaline lakes and Spirulina (Arthrospira) resources in sandy land of Erdos. J Arid Land Res Env 15(4):86-91

Voushak A, Richmond A (1988) Mass production of blue-green alga Spirulina: an overview. Biomass 15:233-247

Vonshak A (1997) Spirulina: growth, physiology and biochemistry. In: Vonshak A (ed) Spirulina platensis (Arthrospira): physiology, cellbiology and biotechnology. Taylor \& Francis, London, pp 43-65

Wu BT, Xiang WZ, Tseng CK (1998) Spirulina cultivation in China. Chin J Oceanol Limnol 16 (Supplement 1):152-157 